\title{
Economía social y vivir bien en el contexto urbano: una experiencia institucional en Medellín, Colombia
}

\author{
Indira Isabel Jiménez-Inchima*, Esperanza Gómez-Hernández ${ }^{\star *}$
}

Candidata a doctora en Ciencias Sociales, Grupo de Estudios Decoloniales e Interculturales, Universidad de Antioquia, Medellín, Colombia. Investigadora visitante Flacso, Quito, Ecuador. Correo electrónico: indira.jime@gmail.com

* Doctora en Educación, Línea Estudios Interculturales, Profesora, Universidad de Antioquia, Medellín, Colombia. Correo electrónico: rubyesperanza@gmail.com, decolonialeintercultural@gmail.com

Recibido: 1 de mayo del 2016

Aprobado: 7 de noviembre del 2016

Cómo citar este artículo: JiménezInchima, I. I. y Gómez-Hernández, E. (2017). Economía social y vivir bien en el contexto urbano: una experiencia institucional en Medellín, Colombia. Cooperativismo \& Desarrollo, 110(25), 55-68. doi: https://doi. org/10.16925/co.v25i110.1763.

\section{Resumen}

Introducción: este artículo presenta los resultados de investigación del estudio realizado en las unidades productivas asociativas que surgen bajo el marco del programa de economía solidaria, desarrollado por el Gobierno municipal de Medellín, con la intención de mostrar el alcance y las limitaciones que este tipo de propuestas tienen para fortalecer otras formas de organización y producción alternativa basadas en la solidaridad, y, en esta misma perspectiva, en el mejoramiento de la calidad de vida de las personas que hacen parte de estas. Metodología: se llevó a cabo un estudio de tipo cualitativo a través de entrevistas directas a profundidad, realizadas a diferentes actores que participan o han participado en el programa de economía solidaria en el Gobierno municipal. Resultados: al seguir afianzado una economía de mercado, prevalece una noción de calidad de vida instrumentalizada que invisibiliza otros sentidos de vida que emergen en consonancia con las concepciones propias del vivir bien del contexto analizado. Conclusiones: siguen existiendo aún grandes desafíos para fortalecer la economía solidaria en Medellín, una economía que esté sustentada en otro tipo de relaciones de producción, intercambio y cooperación, y que fortalezca al sujeto solidario.

Palabras clave: calidad de vida, contexto urbano, economía social y solidaria, programas gubernamentales, vivir bien. 


\title{
Social Economy and Living Well in the Urban Context: an Institutional Experience in Medellín, Colombia
}

\begin{abstract}
Introduction: This article presents the results of the study carried out in the associative productive units that arise in the framework of the solidarity economy program, developed by the municipal government of Medellín. The intention is that of showing the scope and limitations that these types of proposals have to strengthen other forms of organization and alternative production based on solidarity, as well as for improving the quality of life of the people who make part of them. Methodology: A qualitative study was carried out with direct in depth interviews done to different actors that participate or have participated in the municipal government's solidarity economy program. Results: Because a market economy is still being consolidated, an instrumentalized notion of quality of life prevails. This makes invisible other meanings and dimensions of life, which emerge with the conceptions of living well that belong to the analyzed context. Conclusions: Major challenges still persist to strengthen the solidarity economy in Medellín, an economy based on other types of production, exchange and cooperation relations, and that reinforces a solidary subject.
\end{abstract}

Keywords: quality of life, urban context, social and solidarity economy, government programs, living well.

\section{Economia social e viver bem no contexto urbano: uma experiência institucional em Medellín, Colômbia}

\section{Resumo}

Introdução: este artigo apresenta os resultados de pesquisa do estudo realizado nas unidades produtivas associativas que surgem sob o contexto do programa de economia solidária, desenvolvido pelo governo municipal de Medellín, com a intenção de mostrar o alcance e as limitações que esse tipo de propostas tem para fortalecer outras formas de organização e produção alternativa baseadas na solidariedade e, nessa perspectiva, na melhora da qualidade de vida das pessoas que fazem parte destas. Metodologia: realizou-se um estudo de tipo qualitativo por meio de entrevistas diretas a profundidade, realizadas a diferentes atores que participam ou participaram do programa de economia solidária no governo municipal. Resultados: ao continuar consolidando uma economia de mercado, prevalece uma noção de qualidade de vida instrumentalizada que invisibiliza outros sentidos de vida que emergem em consonância com as concepções próprias do viver bem do contexto analisado. Conclusões: continuam existindo ainda grandes desafios para fortalecer a economia solidária em Medellín, uma economia que esteja apoiada em outro tipo de relações de produção, intercâmbio e cooperação, e que fortaleça o sujeito solidário.

Palavras-chave: contexto urbano, economia social e solidária, programas governamentais, qualidade de vida, viver bem. 


\section{Introducción}

Estamos en un contexto de globalización basado en el capitalismo y que se fundamenta, precisamente, en la nociones de desarrollo y progreso, las cuales han conllevado a crisis cada vez más profundas (Escobar, 2007; Marañón-Pimentel, 2012; Quijano, 2012), enmarcadas en el desempleo estructural, el hambre, el cambio climático y la desigualdad, y que no solo repercuten en lo económico, sino también en lo social, cultural, ecológico y humano (Cordero-Ramírez y Ortiz-Ibáñez, 2012). En este escenario, se vienen evidenciando prácticas alternativas a través de la construcción de propuestas colectivas enfocadas en mostrar otros tipos de valores, entre los que se encuentra la solidaridad, la justicia en igualdad, y que desde esta perspectiva proponen una ruptura con los pilares predominantes del modelo de economía de mercado, y, de acuerdo con Coraggio (2011), se enmarcan bajo el paradigma de la economía social y solidaria (en adelante, Ess).

En este sentido, y desde el contexto institucional, en Latinoamérica - en países como Argentina, Uruguay, Brasil, Colombia y, especialmente, en Ecuador y Bolivia-, vienen emergiendo experiencias que desarrollan estrategias establecidas dentro del discurso de la economía solidaria con el objetivo de mejorar la calidad de vida de las personas, a través de programas gubernamentales, enmarcados dentro del diseño y aplicación de sus políticas públicas.

En Colombia, específicamente en Medellín, esto no ha sido la excepción, ante un contexto económico y social con altas tasas de desempleo y subempleo ${ }^{1}$, así como con altos niveles de desigualdad ${ }^{2}$ y pobreza de finales de la década de los noventa y principios de la década del 2000, el Gobierno municipal de la ciudad ha dado impulso a estrategias de intervención desde la perspectiva de la economía solidaria. Esto lo ha hecho a través de los planes de desarrollo 20082011: Medellín es solidaria y competitiva y 2012-2015:

1 Tasa de desempleo de $16,98 \%$ y tasa de subempleo del $30 \%$ en enero del 2003 (DANE, 2008).

2 Coeficiente de Gini de 0,557 en el 2003: cálculos Mesep y el Departamento Administrativo Nacional de Estadística (DANE) con base en la Encuesta Continua de Hogares 2002-2005 y Gran Encuesta Integrada de Hogares 2008-2011.

Nota: este coeficiente es un número entre 0 y 1 , donde 0 corresponde a la perfecta igualdad (todos tienen los mismos ingresos) y 1 es la perfecta desigualdad (una persona tiene todos los ingresos y los demás ninguno).
Un hogar para la vida. También estuvo amparado en las figuras de participación comunitaria como el presupuesto participativo ${ }^{3}$ (en adelante, PP), orientado hacia la generación de autoempleo e ingresos en las diferentes comunas y corregimientos de Medellín.

La economía solidaria se plantea como una forma alternativa de hacer economía (Razeto, 2010), en la cual confluyen otros principios como la reciprocidad, la solidaridad, la complementariedad, y supera las pautas culturales aceptadas por la mayoría de la población que se enfocan en la permanente acumulación de bienes materiales (Acosta, 2012). Aunque no postula una negación de la economía de mercado, sí tiene una orientación crítica y transformadora con respecto a las grandes estructuras y formas de organización que caracterizan la economía contemporánea (Razeto, 2001). Por esa razón, el propósito de esta investigación es presentar los resultados de investigación que en este sentido permitan responder sobre el alcance y las limitaciones de las iniciativas de Ess diseñadas e implementadas desde el Gobierno local en relación, justamente, con la posibilidad que estas tienen de fortalecer otras formas de organización y producción alternativa al modelo económico hegemónico y que en este mismo sentido incidan desde la propia concepción de quienes hacen parte de estas, en lo que ellos determinan como calidad de vida, la cual estará directamente relacionada con sus ámbitos personal, familiar y comunitarios.

El texto se desarrolla de la siguiente manera: primero, se presenta un abordaje teórico general del concepto de la Ess, que abarca sus principales corrientes teóricas, principios y su relación con el buen vivir; segundo, se realiza una contextualización del enfoque y el proceso que desde la política pública en los contextos nacional y local y, en este mismo sentido, viene desarrollando la ess en Colombia y Medellín, en este último caso, desde el programa de economía solidaria; tercero, se presenta la metodología para llevar a cabo la investigación; cuarto, se muestran los resultados y hallazgos de un caso particular del municipio de Medellín a través de las unidades productivas asociativas (UPA) que hacen parte del programa de economía solidaria; y finalmente, las conclusiones.

\footnotetext{
3 El presupuesto participativo surge en la ciudad de Medellín en el 2004 como un instrumento de planificación anual. De acuerdo con la Alcaldía de Medellín tiene el objetivo de dar priorización de las demandas de la ciudadanía, permitiendo un acceso a esta en las decisiones sobre inversiones de la ciudad, desde una perspectiva que busca integrar el desarrollo de la ciudad con la planeación y el desarollo de las comunas y corregimientos (Alcaldía de Medellín, 2005).
} 


\section{Economía social y solidaria y buen vivir: una perspectiva desde América Latina}

En las últimas décadas, diferentes autores se han destacado por desarrollar una perspectiva crítica de la noción idealizada del desarrollo, caracterizada por su enfoque economicista, el cual naturaliza el lucro individual y la explotación de los factores productivos en provecho de la acumulación de capital. Autores como Arturo Escobar (2007), por ejemplo, ubican la idea de desarrollo como un proyecto económico y cultural, desde el cual las sociedades europea y norteamericana fijan parámetros, que, bajo lineamientos occidentales, se enfocan en el crecimiento económico, la lógica del mercado y la explotación de recursos; y el bienestar humano queda sujeto al consumo material y explotación de la naturaleza (Gudynas y Acosta, 2011).

En contraposición, autores destacados en Latinoamérica (Estermann, 2006; Gudynas y Acosta, 2011; Quijano, 2012) rescatan el buen vivir como una propuesta que reacciona y se postula como alternativa al desarrollo, y que se nutre desde diversos campos, desde las tradiciones indígenas hasta las prácticas ciudadanas (Gudynas y Acosta, 2011). El buen vivir propone una vida en armonía con la naturaleza y las personas, $y$, en este sentido, sirve de plataforma en la crítica al desarrollo contemporáneo. De acuerdo con Estermann (2006): "El vivir bien no es riqueza ni pobreza, no es despilfarro ni escasez, no es lujo ni carencia, sino vida en armonía con todos los demás seres, una convivencia intercultural, interbiológica e intergeneracional" (p. 11), es decir, cuestiona la racionalidad del desarrollo actual, su énfasis en los aspectos económicos, la obsesión por el consumo, el mito del progreso continuado, el énfasis convencional de entender el bienestar únicamente como una cuestión de ingresos económicos o posesión material que solo se puede resolver con el mercado, el reduccionismo de presentar el desarrollo como crecimiento económico y el papel de la naturaleza como objeto.

En este sentido, el buen vivir se postula como un camino que busca disociar la calidad de vida, en el cual los valores enfocados van más allá de la simple noción de bienestar, reducido hasta ahora al consumo de bienes materiales (Aguado et al., 2012). Desde este enfoque, la economía deberá tener un rumbo distinto, esto es, su orientación será hacia a la satisfacción de necesidades básicas y a los valores de reciprocidad y complementariedad, que, de acuerdo con
Acosta (2012), "será eficaz para construir relaciones de producción, de intercambio y de cooperación que propicien la suficiencia (más que la sola eficiencia) y la calidad" (p. 70).

Desde esta perspectiva, Acosta (2012) afirma que "el buen vivir como alternativa al desarrollo reclama otra economía" (p. 162), lo que significa una economía que esté fundamentada en la solidaridad, la reciprocidad, integralidad, en otras palabras, otro tipo de relaciones de producción, de intercambio, cooperación, acumulación de capital y ante todo respeto hacia la naturaleza; una economía que cuestione las relaciones antropocéntricas, que supere los patrones culturales heredados y que se enfocan en una lógica económica orientada a la ampliación permanente del consumo en función de la acumulación de capital.

Es importante anotar que el buen vivir no se plantea como una propuesta esencialista, pues, aunque cuente con un anclaje histórico en el mundo indígena $^{4}$, tiene aspectos que convergen, pero que también se contraponen dependiendo de los contextos culturales, históricos y ambientales (Gudynas y Acosta, 2011) que se habiten. El vivir bien, buen vivir, estar bien "si bien pueden compartir cosmologías afines, también muestran un carácter diverso, una necesidad de entender porque la vida en un territorio es expresada de muchas maneras y con variados lenguajes" (Gómez, 2014, p. 133).

Para el contexto de Medellín, el buen vivir surge dentro de los discursos que emergen de manera libre dentro del ámbito de la cotidianidad vecinal, y que en este sentido abre la posibilidad de entender otras maneras de ver el mundo, que surge a partir de los relacionamientos que se establecen en la vida, la vecindad comunal y el medio natural, los cuales son atravesados por valores como la solidaridad, complementariedad y la solidaridad (Gómez et al., 2009). De igual manera, en el ámbito comunitario, el buen vivir - de acuerdo con el contexto- estará relacionado con la capacidad de vivir gracias a las redes comunales que se crean y que garantizan tanto el cuidado como la armonía y que abarcan todas las esferas de la vida (Gómez et al., 2009). Es un espacio comunitario constituido por los lazos de reciprocidad, desde una dimensión socioeconómica, donde coexiste un ideal de economía distinto.

En consecuencia, es desde la economía solidaria que surge la necesidad de replantear el enfoque de

\footnotetext{
4 Conocido como Sumak Kawsay/Sumaq Qamaña, que traduce "buen vivir /vivir bien".
} 
calidad de vida que hasta ahora solo se preocupa por la productividad y el consumo, por la idea de una buena vida construida a partir de la posesión: "Más tengo, más feliz soy y más me reconoce la sociedad" (Coraggio, 2011, p. 4). En este sentido, de acuerdo con Razeto (2006), "plantea la exigencia de la reflexión sobre las dimensiones reales del ser humano y la inclusión en el análisis, de los aspectos político, social y cultural" (p. 7), con el fin de orientar la configuración de organizaciones humanas de carácter alternativo desde una lógica más humana del individuo.

Esta economía solidaria - que replantea y pone en cuestión las relaciones económicas desde parámetros distintos al máximo beneficio- parte de entenderse como una nueva forma alternativa de hacer economía, postula a la solidaridad inmersa en todo el proceso económico, tal y como lo plantea Razeto (1999), "opera y actúa en las diversas fases del ciclo económico: en la producción, circulación, consumo y acumulación; ello implica producir con solidaridad, distribuir con solidaridad, consumir con solidaridad, acumular y desarrollar con solidaridad" (p. 3). Se trata entonces de maneras alternativas de hacer economía que estarían encaminadas no solo a beneficios individuales, sino a beneficios colectivos enfocados hacia una orientación fuertemente crítica y decididamente transformadora respecto de las grandes estructuras y los modos de organización y de acción que caracterizan la economía contemporánea.

\section{Economía social y solidaria: una mirada desde las políticas públicas nacionales y locales}

En el caso de Colombia, la economía social y solidaria se enmarca dentro de un conjunto de planteamientos definidos desde el cooperativismo, que inicia en 1931 con la expedición de la Ley 134. El cooperativismo surge desde las propuestas socialistas ancladas a los movimientos sindicales que defendían los intereses de los trabajadores. En 1986, se adopta el concepto de economía solidaria con el Decreto 2536 del 4 de agosto que dio vida al Consejo Nacional de Economía Solidaria y lo reconoce como un sector de gran importancia en el entorno económico nacional. “En 1988, con la Ley 79 , se organizan las formas solidarias del cooperativismo, asociaciones mutuales y fondos de empleados", es así como hasta 1999, la "supervisión de las entidades de economía solidaria fue adelantada por el Departamento Nacional de Cooperativas - Dancoop-, la institución del Gobierno encargada de definir la política para las formas solidarias de organización en particular para el sector cooperativo. Esta entidad tenía a su cargo la planificación de sus políticas, la ejecución de programas y proyectos, y, a su vez, el ejercicio del control de gestión de estas (Superintendencia de la Economía Solidaria, 2016).

Con el objetivo de dotar al sector cooperativo de un marco propicio para su desarrollo como parte fundamental del cooperativismo, se estipula la Ley 79 de 1998. Así mismo, la Ley 454 de 1998 determina el marco conceptual de la economía solidaria así: "Sistema socioeconómico, cultural y ambiental conformado por el conjunto de fuerzas sociales organizadas en formas asociativas identificadas por prácticas autogestionarias solidarias, democráticas y humanistas, sin ánimo de lucro para el desarrollo integral del ser humano como sujeto, actor y fin de la economía" (p. 1).

Igualmente, al Departamento Administrativo Nacional de la Economía Solidaria (Dansocial) se le asignó la función de formular, orientar y desarrollar políticas para el fomento y fortalecimiento de organizaciones solidarias; sin embargo, en el 2011, esta entidad $^{5}$ se transforma en la Unidad Administrativa Especial de Organizaciones Solidarias, que se adscribe al Ministerio de Trabajo. Igualmente, se crean la Superintendencia de la Economía Solidaria (Supersolidaria) con la finalidad de vigilar y controlar, y el Fondo de Garantías para las Cooperativas Financieras y de Ahoro y Crédito (Fogacoop) para dar confianza y fortalecimiento a la actividad financiera cooperativa.

\section{Contexto local}

Para hablar de la Ess en Medellín, es necesario abordar su historia reciente, enmarcada por la problemática del narcotráfico, vivida intensamente casi por una década (1985-1993), y que reorientó en gran medida la estructura social, el comportamiento cultural y en general la economía de la ciudad. Esto se encontraba unido a una crisis socioeconómica vivida entre 1995 y comienzos de este siglo, en la cual Medellín llegó alcanzar niveles de desempleo por encima del 20\% (1999).

Aunque luego de esta crisis económica de finales de los noventa, la ciudad tuvo una recuperación económica y social, para el 2003 la ciudad continuaba mostrando condiciones difíciles en el contexto socioeconómico que se hacían evidentes tanto en

Con el Decreto Nacional 4122 del 2 de noviembre de 2011 
materia de desempleo (14\%), en la cual las mujeres y los y las jóvenes eran los más afectados (con porcentajes del 50\%, 35\% y $40 \%$, respectivamente), como en la baja inclusión social (altas desigualdades que se dan por razón de estrato socioeconómico, por género, por grupo etario, por etnia, por convicciones religiosas y hasta por preferencias sexuales); en desigualdad, reflejada en indicadores como en el índice de Gini de 0,56, y una percepción ciudadana ( $58 \%$ de los encuestados) de que la calidad de vida en Medellín seguía igual o había empeorado en el estrato bajo (2001-2003) por nombrar algunos.

Ante esta problemática que enfrentaba la ciudad en materia de empleo, generación de ingresos y desigualdad, Medellín ha planteado diferentes metodologías para la participación democrática a través de los planes de desarrollo. En estos, se da la construcción de escenarios deliberativos en los que es posible la formulación de diagnósticos, la priorización de las inversiones y la concertación del desarrollo local.

Es así como, para el 2004, en el marco del surgimiento de presupuesto participativo, el gobierno de Sergio Fajardo implementa el plan de desarrollo: $\mathrm{Me}$ dellín: compromiso de toda la ciudadanía (2004-2007). Con esto pretendía dar impulso, a través de la línea 4: "Medellín productiva, competitiva y solidaria", a la estimulación de distintas formas de asociatividad y economía solidaria. En este sentido, se posibilita la participación en los encadenamientos productivos de la región con el objetivo de lograr "la creación de más puestos de trabajo estables y productivos, con los impactos sociales que esto implica sobre las poblaciones más vulnerables" (Alcaldía de Medellín, 2011, p. 127)

Bajo esta misma perspectiva, para el siguiente periodo (2008-2011), Alonso Salazar implementa el plan de desarrollo Medellín es solidaria y competitiva bajo la línea 3: "Desarrollo económico e innovación". Este buscaba seguirle haciendo frente a la problemática del empleo, que, aunque mostraba una recuperación (11,9\% en el 2007), se veía opacada por una desmejora en la calidad del trabajo reflejada en la tasa de subempleo (20\% en 2005 y $27 \%$ hacia finales del 2007). En un marco general, el diseño de esta línea estaba orientado a la búsqueda del afianzamiento de una estrategia que asumiera el desarrollo de la ciudad desde una perspectiva de desarrollo humano integral, abordando problemas de empleo, autoempleo y emprendimiento, en el cual se tuvieran en cuenta factores diferenciales de acuerdo con las necesidades específicas de las poblaciones vulnerables y se fortaleciera una plataforma socioeconómica atractiva para la inversión de la ciudad (Alcaldía de Medellín, s. f.).
En este contexto, se da origen al Acuerdo 41 del 2011 "por medio del cual se adopta la política pública para la economía social y solidaria en el Municipio de Medellín”, y, en apoyo con el Acuerdo 43 de 1996 "por el cual se crea e institucionaliza la planeación local y el presupuesto participativo en el marco del Sistema Municipal de Planeación", orientan la Unidad de Economía Social y Solidaria bajo la adscripción de la Subsecretaría de Fortalecimiento y Creación Empresarial, la cual ha venido diseñando programas que han impulsado la creación de empresas sociales y solidarias, como es el caso del Programa de Economía Solidaria de la Secretaría de Desarrollo Social ${ }^{6}$.

Así mismo, para el periodo de Aníbal Gaviria (2012-2015), se impulsa el plan de desarrollo Un hogar para la vida. Este, bajo el programa de emprendimiento y desarrollo social y solidario, continuó apoyando las iniciativas pertenecientes a la economía solidaria, con el fin de generar empleo e ingresos en las personas, enfocado a la disminución de la pobreza y las desigualdades.

\section{Descripción del programa de economía solidaria}

Este programa viene desarrollando, desde el 2008, prácticas de apoyo a la economía social y solidaria, y otras formas asociativas que, a través de recursos priorizados por la comunidad en planeación local y pP, con el claro objetivo de crear y fortalecer las unidades productivas como dispositivo para la generación de autoempleo e ingresos en las diferentes comunas y corregimientos del municipio de Medellín (Alcaldía de Medellín, 2011).

El objetivo fundamental de este programa (vigente durante 2008-2011) era fortalecer la cultura del emprendimiento y el empresarismo social y solidario mediante el desarrollo de iniciativas empresariales asociativas, definidas en seis ejes principales:

- Apoyo a la economía social y solidaria

1. Promoción y fomento de buenas prácticas de la economía asociativa y solidaria.

2. Apoyo y acompañamiento al desarrollo empresarial mutualista.

3. La educación alrededor de la economía solidaria.

6 Denominado para la vigencia 2012-2015, "Programa de Emprendimiento y Desarrollo Empresarial Social y Solidario", adscrito a la Secretaría de Desarrollo Social. 
- Apalancamiento de la economía social y solidaria en el marco de planeación local y presupuesto participativo

1. El fortalecimiento de UPA de economía solidaria.

2. Desarrollo e implementación de nuevas formas asociativas, como trueque, los circuitos solidarios, los emprendimientos sociales.

3. Fortalecimiento de organizaciones de segundo grado.

El apoyo a la economía social y solidaria se refería a la capacitación y formación en este tema con el fin de construir bases para generar desde ahí una cultura solidaria en la cual se consolidaran habilidades administrativas para el empresarismo solidario desde diferentes enfoques. En relación con el apalancamiento de la economía social y solidaria, se vinculaba especialmente con el acompañamiento a las UPA, con el objetivo, de acuerdo con el Gobierno municipal, de fortalecer el tejido empresarial de las comunas y corregimientos de Medellín mediante la generación de ingresos y empleo dignos bajo fundamentos solidarios.

Las UPA podían estar conformadas por un grupo de mínimo cinco personas pertenecientes a la comuna, las cuales se unen para desarrollar una actividad económica sin ánimo de lucro. De acuerdo con la Alcaldía de Medellín (2011), su actividad estaría enfocada en la producción de bienes y servicios competitivos en el mercado que permitan la generación de ingresos para la reinversión o la distribución equitativa de los excedentes económicos generados y la creación de empleos dignos. Hacen parte personas que busquen la autosostenibilidad, la sustentabilidad y el beneficio para sus asociados y la comunidad de la cual hacen parte (p. 13). La selección de las UpA para apoyar se realiza a través de un concurso que, por medio de una entidad - denominadas operadores - y un sistema de puntos, escoge las mejores iniciativas. El objetivo de los operadores era justamente llevar la gerencia y asesoría para el acompañamiento empresarial de las iniciativas y proyectos económicos solidarios.

Así mismo, para participar, las iniciativas debían contar con un cierto número de requisitos, por ejemplo: que la comuna a la que pertenezca si haya

Para la vigencia del 2011, los operadores contratados fueron Fedemutuales, unión temporal conformada por Fomentamos y Confiar. Además de la uT Cisp Católica del Norte, Corprogreso, UT Ikala Mademo, Universidad de Medellín Portafolio Verde y Cemped. En el 2013, Creame, Incubadora de empresas, fue la entidad encargada. priorizado recursos del PP para este eje; mínimo el $80 \%$ de los miembros de la base asociativa deberían vivir en la comuna a la cual pertenecen los recursos; así mismo, la base asociativa no podía estar constituida por más de dos personas que sean familiares.

\section{Metodología}

Teniendo en cuenta los cuestionamientos que convocan este estudio, se optó por una investigación cualitativa en cuanto que esta nos permite trabajar con el universo de significados, motivos, aspiraciones, creencias, valores y actitudes (Souza, 2003) de los actores relacionados con las diversas experiencias de economía solidaria de Medellín. También se adoptó un método de estudio de caso, ya que nos permitía realizar una investigación empírica dentro de un contexto de la vida real (Yin, 1994) de tipo descriptivo, orientada a la investigación de la comprensión en profundidad del objeto de estudio (EES), desde un programa de economía solidaria municipal, afianzado con otras investigaciones realizadas en Medellín sobre el programa de economía solidaria, y que en este sentido nos permitieron hacer relaciones y comparaciones que afianzaron nuestros resultados de investigación (Álzate y Betancur, 2014; Amaya y Colón, 2013).

Para el análisis de la información, se tomó en cuenta el sistema categorial, que surgió de la articulación entre la información construida por las personas que hacen parte del programa de economía solidaria y referentes conceptuales. Teniendo en cuenta lo anterior, se procedió a identificar los representantes del Gobierno que estuvieron a cargo de la implementación del programa, para posteriormente realizar la aplicación de entrevistas semiestructuradas.

Para la identificación y selección de las UPA, se tuvo en cuenta la base de datos otorgada por la Secretaría de Desarrollo, la cual relacionaba 144 unidades, de las cuales fueron seleccionadas $20^{8}$, ubicadas en las comunas 1 (Popular), 2 (Santa Cruz), 3 (Manrique) y 4 (Aranjuez) de Medellín. Se escogieron estas comunas pues eran los territorios donde se concentraban el mayor número de UPA en la ciudad, lo que nos permitía tener una perspectiva más amplia y precisa del objeto de estudio.

Las entrevistas realizadas se basaron en una guía de preguntas adaptadas en función de la función y las actividades que desarrolla el interlocutor. Para la

8 En del 2016, 15 de estas unidades siguen activas. 
selección de UPA, se contó con el apoyo de los centros de desarrollo empresarial zonal (Cedezos) ${ }^{9}$, que ayudaron a identificar las unidades que ellos consideraban exitosas (15 UPA). Así mismo, se tuvieron en cuenta para el estudio cinco UPA que en algún momento habían alcanzado cierto grado de reconocimiento en el programa, pero que posteriormente renunciaron o se consideraron inviables. El trabajo de campo se realizó entre enero y junio del 2014, y, para ello, fue necesario el desplazamiento a sus casas o al lugar donde funcionaba la UPA. Es importante anotar que la realización de las entrevistas resultaron ser una vía para conocer los barrios, las personas y las relaciones que se tejen no solamente con los otros miembros de la UPA, sino también con sus vecinos y su familia.

\section{Resultados}

\section{Una propuesta empresarial orientada a la generación de ingresos y empleo}

En primera instancia, es importante partir del enfoque y los objetivos con el que se da origen y que motivan el diseño y la implementación del programa de economía solidaria por parte de la administración municipal de Medellín. De acuerdo con la información recolectada y las fuentes secundarias que lo describen, se pudo determinar que su enfoque estaba orientado hacia la promoción de ingresos y empleo, que, por medio de la creación de las UPA, buscaba la superación de la pobreza y, en ese mismo sentido, el mejoramiento de las condiciones de vida de los ciudadanos de Medellín.

Desde estos planteamientos, la creación de este modelo respondía a la necesidad de impulsar la creación de unidades de trabajo como alternativa de solución al desempleo, afianzada en los recursos del PP. Sin embargo, desde su implementación, el modelo no ha logrado consolidarse como una alternativa viable para fortalecer la economía solidaria como posibilidad de transformación social en la ciudad, ya que, aunque teóricamente se sostiene en un paradigma teórico alternativo, en la práctica se distorsiona siguiendo el enfoque de la economía tradicional.

\footnotetext{
9 Son un espacio físico - adscritos a la Alcaldía de Medellínubicados en las diferentes comunas de la ciudad con el propósito de poner a disposición del tejido empresarial y social de estos sectores, servicios en pro del fomento y desarrollo del empresarismo. Sus servicios se centran en: apoyo al empresarismo en el territorio y fortalecimiento de las actividades empresariales existentes.
}

En palabras de un funcionario de la administración municipal, quien participó en el diseño del programa de 2008 a 2011: "Lo que pasa es que como es un recurso público no puedes beneficiar una persona, tienes que beneficiar un colectivo por eso el enfoque solidario. De todas maneras era con la mirada empresarial, de que ganes te sostengas en el mercado" (entrevista, 20 de junio del 2014) ${ }^{10}$. Lo anterior concuerda con otras investigaciones llevadas a cabo en diferentes contextos latinoamericanos (Castelao, 2013; Murchio, 2012), en las cuales se ha podido encontrar que dichas propuestas más allá de estar orientadas hacia la búsqueda de un fortalecimiento de la cultura solidaria, han estado dirigidas a la generación de empleos e ingresos, sin una perspectiva de largo alcance que tenga un impacto importante en la redistribución de los ingresos o el fortalecimiento de las empresas autogestionadas.

Es por estas razones que se tiene un profundo carácter cuantitativo en el momento de medir la viabilidad y, en este sentido, los resultados de las UPA. Estos estaban medidos por indicadores de resultado que se enfocaban en el cumplimiento de parámetros cuantificables así: ingresos (medido por el promedio mensual por ventas de los últimos seis meses); empleo generados; base asociativa consolidada (que garantizara la participación activa de mínimo cinco asociados); estructura empresarial adecuada (nivel de formalización); mercado para los productos o servicios, producción (se debían presentar los listados de clientes potenciales y efectivos, reporte de visitas, llamadas a clientes, estrategias de promoción y de servicio); comercialización o prestación del servicio de manera permanente; reinversión de los remanentes en la unidad productiva o en responsabilidad social comunitarias, y finalmente la meta era lograr sostenerse sin apalancamiento de recursos públicos. Las unidades productivas asociativas exitosas estaban caracterizadas por cumplir mínimo cinco de los indicadores de sostenibilidad y en las cuales siempre deben concurrir: ingresos, empleo y base asociativa consolidada (Alcaldía de Medellín, 2013).

De acuerdo con los indicadores de resultado, el programa tenía serias limitaciones para cumplir con sus objetivos propuestos, ya que se evidencian aún grandes obstáculos dentro de los que se encontraba un alto grado unidades inviables (61 unidades para el periodo 2010-2011, proyectos inconclusos por

\footnotetext{
10 Se protege su nombre para respetar la privacidad de sus opiniones.
} 
diferentes dificultades (57 renuncias) en comparación con las UPA apoyadas en ese mismo periodo.

En este sentido, la Administración Municipal (Alcaldía de Medellín, 2011) afirmaba que entre las problemáticas más comunes se encontraban la falta de visión empresarial de las personas que participaban en el proceso, sumada a falencias en la financiación de las iniciativas productivas asociativas, ya que contaban exclusivamente con recursos del programa de planeación local y pp, lo cual hacía que los procesos se desarrollaran en tiempos de ejecución ajustados y con periodos muertos que no permiten seguir las dinámicas propias de una empresa ni del mercado.

Lo anterior resulta reiterativo desde las palabras de un funcionario de la administración municipal:

Había que fortalecer las capacidades para que sean empresarios, usted ve a un señor que lleva una junta de acción comunal haciendo un trabajado comunitario difícilmente lo pone en el rol de empresario porque no es que usted le ponga el rol, uno lo tiene y se potencia, es un error que uno comete que cometimos muchos en la alcaldía de Medellín con eso, y es pensar que los líderes son sinónimos de empresarios, ahí vamos perdiendo (entrevista, el 14 de junio del 2014).

\section{Heterogeneidad en las perspectivas del enfoque solidario desde su aplicabilidad}

Cabe destacar que, en general, las UPA entrevistadas empezaron su actividad en el 2008, pero algunas de ellas se formalizaron en el 2010. Partiendo del enfoque del programa, se vio de manera frecuente cómo dentro de los discursos de las personas que hacen parte de las UPA, el aspecto empresarial estaba presente como una constante al entender el programa, es decir había dificultades en el modo de comprensión del modelo solidario desde el discurso.

Del trabajo de campo realizado, se pudo constatar que existe heterogeneidad en el alcance que desde estas prácticas ejerce la economía solidaria de acuerdo con sus principios. En primera instancia, las unidades empezaron con una conformación de la base asociativa de 20 a 25 personas, pero, con el paso del tiempo y al no ver los beneficios especialmente económicos a corto plazo, se retiraron, manteniéndose en el tiempo solo los creadores de la idea de negocio, quienes en su mayoría son los representantes legales actuales. Ellos son líderes comunales que cumplen un papel importante en la comunidad y que desempeñaban una función activa, específicamente, en la comisión económica y han visto en estos programas una opción, una oportunidad para crear su propio negocio.

Los asociados eran casi siempre vecinos y amigos de la misma comuna; sin embargo, esta asociación se hacía más como un requisito: era el representante legal quien tenía el control y el mando de la unidad. En varios casos los integrantes de la base asociativa eran empleados y no tenían un vínculo real con el ejercicio de la UPA. En este mismo sentido, la formación en economía solidaria era aceptada más como un requisito para acceder a los beneficios del programa de economía solidaria y no con la intención de hacer parte de un proyecto político transformador y que postule nuevos frentes. Al respecto, la representante lega ${ }^{11}$ de la UPA afirma:

¡Ay!, eso sí es más mentiras, pues yo en ese cuento no creo, ni nunca he creído, yo no creo que se junten cinco personas que no se conocen, porque así son las reglas, yo no creo que yo me pueda unir con cinco personas que no conozco, que eso es lo que ha pasado con todo lo de economía solidaria, [...] la base social que yo tenía hace cuatro años, no es la misma en este momento, como te juntan con cinco personas que no conoces [...] es complicado, para mí lo de economía solidaria está mal enfocado, pero, esa es la economía que la $\mathrm{Al}$ caldía montó, y ahí si no podemos hacer nada, hay que seguirles la corriente no (entrevista, mayo del 2014).

Sin embargo, teniendo en cuenta que desde este programa institucional transitan distintas experiencias, es importante destacar que, por otro lado, se encontraron experiencias que han logrado mantenerse en el tiempo; igualmente, aunque su base asociativa se modificaba - con disminución y rotación importante en el tiempo-, y aunque no tuvieran claro desde el discurso el significado de la economía solidaria, han logrado llevar a cabo prácticas solidarias en diferentes grados de aplicación.

Es importante anotar que, en estos casos, las personas que hacían parte de estas unidades tienen la percepción de que más allá de un ejercicio económico comprenden que su unidad productiva tiene un sentido más amplio que trasciende, ya sea desde el ámbito cultural, social o ecológico, y que, en esta perspectiva, lograban generar el fortalecimiento de los vínculos sociales, pero que, sin embargo, entraban en puja

11 Se omite el nombre por solicitud de la persona entrevistada. 
constante con los lineamientos y mediciones que desde el programa se planteaban, pues sus parámetros entran en contradicción, y en este mismo sentido, sus mediciones no cumplían con los indicadores cuantitativos de la administración municipal. Al respecto, la representante legal de la UPA Codesfam afirma:

Pues ellos como que lo que más se están fijando es cuántos empleos dignos estás generando vos en esa unidad productiva, pero esas historias de vida no las ven, y esa ganancia es mejor que el dinero, para mí, y ahí es donde ellos deberían entrar hacerle el apoyo, o sea, no mirarlo a uno que porque no está produciendo, y de pronto yo diría que ellos se fijan mucho porque es el cuento que hay muchas UPA que mientras que haya presupuesto participativo funcionan, se acaba y se cierran, dicen va a haber presupuesto participativo, y ahí mismo vuelven y abren, y a nosotros eso no nos pasa, nosotros, haya o no haya, siempre estamos, en este momento los cursos nos están generando el arriendo y los servicios, listo pare de contar, vendrán tiempos mejores y ahí estamos (entrevista, 12 de abril del 2014).

Es, en este sentido, que se muestra que el programa en sus lineamientos y perspectivas no tiene un enfoque, en el sentido que Razeto (1999) propone, como apuesta transformadora, es decir, no estaba enfocada para que, desde el plano de la producción, prevaleciera el trabajo sobre el capital; o que, desde la distribución, se fortalecieran la reciprocidad y la cooperación a través de redes o circuitos solidarios o que desde el consumo, existiera un énfasis hacia el cambio de cultura actual de los constructos de satisfacción de necesidades. Por el contrario, su enfoque estaba orientado a asociatividades inmediatas, con el fin de producir un bien o servicio que debía competir en un mercado y que en muchos casos no tenía en cuenta sus saberes, ni las lógicas cotidianas en el ámbito personal, familiar y vecinal de quien hace parte de estas propuestas.

\section{La economía solidaria como un modelo alejado de las realidades sociales}

Cabe señalar, además, que en los discursos reconocidos por las personas de las UPA el aspecto empresarial está presente como una constante de los programas y de sus unidades. Destacan la economía solidaria como un modelo difícil de poner en práctica teniendo en cuenta el contexto económico y social; lo asociativo, se ve como un frente de ruptura por la falta de apropiación de compromiso con la UPA, pero principalmente por la necesidad de obtener un ingreso fijo que le permita suplir sus necesidades de corto plazo. Al respecto, Paula Cano, representante legal de la unidad productiva Arte en mis Manos, comenta:

Cuando vamos al hecho a lo real no todos colaborábamos, somos tres o cuatro los que hacemos, y si somos diez, de esos diez no hacen sino cinco. Es un concepto difícil [...] todos tenemos que aportar, pero quien le va a pagar el arriendo a aquella, aquella también necesita pagar servicios, y esta UPA no tiene para darle eso hace que se desvincule ese grupo, se deben ir a trabajar a otro lado (entrevista, 3 de marzo del 2014).

Las personas entrevistadas destacan la economía solidaria como un discurso propuesto desde el Estado, pero que no tiene un acercamiento con la realidad del quehacer de sus unidades, además porque sigue existiendo una dependencia muy fuerte de los asociados por el trabajo asalariado. Es el dinero lo que sigue marcando las relaciones de estos procesos, dejando de lado las relaciones de reciprocidad o solidaridad. Julio Marín, representante legal de Cooperativa Multiactiva Unidos por Colombia y Una Antioquia Grande, señala:

Falta mucho, es que ese proceso es muy lento y realmente trabajar en equipo y llevar lo que es la economía solidaria y trabajar sin ánimo de lucro. Muchas personas no lo entienden y no lo aceptan. Ese ha sido el gran atraso de esto, no solamente en la mía sino que yo veo que en Colombia los colombianos somos como escépticos, no le apuntamos a trabajar en equipo mucho porque hasta ahí. Cuando se trate de hacer el equipo o decir que yo no voy a trabajar, yo consigo un asociado y el busca un beneficio más propio que comunitario ante ese aspecto es muy complicado (entrevista, 16 de mayo del 2014).

\section{Percepción de incidencia en la calidad de vida}

En las entrevistas se encontraron respuestas ambivalentes sobre la percepción de la incidencia de pertenecer al programa en su calidad de vida. En primera instancia, los entrevistados aseguraban, por una parte, que su calidad de vida - o más bien desde su propio discurso, su vida- no había mejorado. Esto se explica por la fuerte presión, producto de las 
dinámicas de formalización, las capacitaciones y el alcance de los objetivos que son definitivos en el momento de conseguir recursos para su emprendimiento; así como la sensación de abandono por parte del Estado, al no contar con ellos para las contrataciones; además de la formalización de los procesos que exige el mercado para poder ser competitivos (impuestos, certificaciones, permisos, aspectos legales).

Así mismo, a pesar de que el pp tenía como objetivo dar a las comunidades poder de decisión, tomando un papel más activo y convirtiéndose en sujetos con poder en la destinación de los recursos públicos. Como afirman Gómez et al. (2009), "surge ligado a la urgencia de la participación ciudadana como estrategia para hacer posible que los programas de desarrollo lograran implementarse de manera efectiva y combatan la pobreza, así como a la imperativa necesidad de legitimidad estatal mediante el cogobierno entre Estado y ciudadanía” (p. 66). Para este caso, la priorización de los recursos en las comisiones económicas de presupuesto participativo los había enfrentado a conflictos barriales, políticos y de diversas índoles que eran ocasionados en algunos casos por la sensación de desconfianza y malestar entre los vecinos.

Por otra parte, las personas entrevistadas coincidían en afirmar que su calidad de vida había mejorado, en el sentido que habían logrado obtener no solo un beneficio en torno a su vida propia y su familiar, sino que a nivel comunitario lograban fortalecer sus relaciones sociales a través del ejercicio de la UPA. A pesar de que, en muchos casos, no se cumplían con los parámetros de medición de la administración municipal - entrando en contraposición con los intereses y perspectiva del programa-, conseguían generar espacios de fortalecimiento de la comunidad, dirigidos a mujeres, jóvenes, en general, a personas desempleadas. Esto abría una puerta hacia una oportunidad, no solamente entendida como una fuente de ingresos, sino, además, como la oportunidad de generar lugares de esparcimiento, de capacitación, de escucha y de fortalecimiento de espacios en pro de relacionamientos sociales y culturales.

Igualmente, entre esas transformaciones, se encontró una incidencia, especialmente en las mujeres. El tema de las capacitaciones que han recibido les ha permitido desarrollar actitudes que, aunque no siempre han funcionado desde el enfoque instrumental de la productividad de la UPA, sí les ha dado más seguridad y liderazgo en su comunidad y su entorno familiar, tal y como lo señala Alba Luz Narváez, representante legal de la UPA Artesanos de Colombia:
Sí porque como conozco de leyes, conozco más derechos, pues yo ya los defiendo: a mí nadie me va gritando así como así, a mí no nadie me va a decir es que usted no va por allá porque yo digo que no. Ya tengo argumentos y conozco mis derechos como mujer, entonces, yo ya tengo ese empoderamiento de mujer para defender mi situación, y las de otras mujeres, aquí podemos conversar con las compañeras, oírnos y darnos consejos (entrevista, 3 de marzo del 2014).

De la misma forma, el trabajo era concebido como un espacio de dignificación, resultado del ejercicio en la UPA. Al respecto, los entrevistados coincidían en decir que se sentían orgullosos de su trabajo, utilizan constantemente en sus discursos las palabras "felicidad", "realización", "motivación". Lo anterior es coherente con la investigación de Alzate y Betancur (2014) que afirma que "existe una fuerte coincidencia entre las UPA, de la persistencia en el trabajo como una forma de reconocimiento social y dignificaciones como seres humanos que aportan a las comunidades donde ellos se acentúan" (p. 12). En general los entrevistados reafirman las buenas relaciones de la base asociativa, que parte de un ambiente tranquilo, de respeto y de esparcimiento en el quehacer productivo, lo que coincidiría con la propuesta de Razeto (1993), según la cual en la economía solidaria el trabajo opera como medio principal para desarrollar las potencialidades que le permiten controlar sus condiciones de vida.

Otros factores de los actores de las UPA que reconocieron haber logrado son el liderazgo comunitario y la valoración social en el barrio. Un reconocimiento de cómo, aunque en principio estuvo enmarcado por la desconfianza y el conflicto con las personas del barrio, con el tiempo han logrado alcanzar una legitimación del ejercicio de la UPA y ser un referente de éxito en la comunidad.

Lo anterior se puede comparar con la investigación de Alzate y Betancur (2014), justamente porque con relación al mejoramiento en la calidad de vida con el programa, partiendo de una escala de valoración de 1 al 5, el 7\% de los encuestados calificaron en 5; el 22\%, en 4 ; el $36 \%$, en 3 ; el $33 \%$, en 2 , y solo el $1 \%$, en 1 . De acuerdo con los resultados, las personas aseguraban que su calidad de vida había mejorado, debido al logro en valores afectivos y espirituales: "Los encuestados manifestaron que este proyecto les ha permitido encontrar un nuevo sentido de vida, crecer como personas, desarrollar mayor sentido de pertenencia y empatías para el trabajo en equipo, mantenerse unidos y a 
la vez generar ingresos para atender la familia; asimismo expresan que se sienten apoyados, útiles y felices" (Alzate y Betancur, 2014, p. 121). Desde esta perspectiva, las personas habían logrado, a través de sus unidades y en conjugación con sus valores de solidaridad de la vida cotidiana, beneficios que van más allá de lo económico y que, en este sentido, aportaban a lo que ellos consideran el vivir bien, aunque entren en contradicción con las perspectivas utilitaristas del programa en cuestión.

\section{Conclusión}

De acuerdo con los resultados, se puede apreciar un caso práctico que, desde el contexto institucional, ha pretendido diseñar y poner en práctica experiencias bajo el discurso de economía solidaria, en el contexto urbano de Medellín. Ante la pregunta sobre los alcances y las limitaciones de estas propuestas que se enmarcan desde la economía solidaria, al menos desde esta experiencia en Medellín, siguen presentándose importantes desafíos para lograr a un nivel más amplio una transformación social que consolide la economía solidaría en Medellín. Lo anterior en razón a que dichas estrategias, a pesar de que se postulen desde la economía solidaria, resultan siendo un mecanismo para fortalecer el espíritu emprendedor y competitivo, enmarcadas bajo el mercado, como un único camino posible. Por lo tanto, están lejos de lograr fortalecer la cultura solidaria en la región.

Así mismo, se encontró que los recursos del PP han jugado un papel fundamental en la reorientación de los fundamentos y los intereses de la economía solidaria, pues han traído consigo conflictos políticos en la comunidad en la perspectiva de consecución de recursos.

A pesar de que el enfoque del programa se orientaba a la medición de la viabilidad de las UPA en términos cuantitativos, se detectó que en algunas experiencias con racionalidades económicas distintas se ha conseguido desde el quehacer de la UPA y en una mezcla - a veces, en conflicto- con el enfoque económico del programa generar transformaciones que no están dentro de los parámetros instrumentales de la calidad de vida, y que, en ese sentido, están más relacionados con sus buenos convivires o vivir bien de su comunidad. En esa perspectiva, están vinculados con relacionamientos más anclados a valores como el reconocimiento, el empoderamiento, liderazgo comunitario y cohesión social, que hacen parte del constructo del sujeto solidario que lo caracteriza.
Sin embargo, lo que resulta de esta investigación es la inquietud sobre la posibilidad que tienen estas iniciativas que surgen desde el Gobierno municipal en lograr impulsar y fomentar la economía solidaria, $y$ en este sentido consigan ser una propuesta transformadora en relación con el modelo económico imperante, que tenga como eje la solidaridad en la vida social, pues, de acuerdo con los resultados de la investigación, resulta más como una apropiación de denominaciones, que en realidad son utilizadas como estrategias para contrarrestar las cifras de desempleo y de desigualdad en la ciudad, con propuestas que denominadas como "alternativas" solo resultan en ejercicios que siguen bajo la misma lógica empresarial y competitiva. Como afirma Razeto (1999), "la economía de solidaridad no es negación de la economía de mercado; pero tampoco es su simple reafirmación" (párr. 24), más allá de que verdaderamente la solidaridad y la equidad sean una propuesta que intente enmarcar una economía que sea capaz de distribuir sus beneficios entre el conjunto de su población y que tenga en cuenta las necesidades y las concepciones propias de las comunidades.

Es así como surgen varios cuestionamientos sobre la incorporación en el discurso y el diseño de programas públicos en nombre de la economía solidaria. ¿Será que, como afirma Coraggio (2011), "son acaso retoques cosméticos a la política social asistencialista o indican un reconocimiento de que la economía no se limita al sector empresarial privado? (p. 37). Se insertan a las comunidades de manera utilitarista al mercado, forzándolas a adoptar un comportamiento empresarial, que significa llevar a estructurar su pensamiento desde la lógica del mercado, reforzando la lógica del emprendimiento, no solo como dispositivo de control, sino como proyectos que desde lo emotivo generan en el sujeto la noción de responsabilidad de sus propios fracasos.

Desde esta perspectiva, es imperante desde el diseño y la ejecución de políticas públicas "reconocer los elementos de esas racionalidades alternativas y las particularidades de las experiencias de solidaridad económica, de manera que desde las políticas sean realmente potenciadas" (Marañón y López, 2014, p. 119). Se logran desarrollar e impulsar las dinámicas solidarias que existen, en este caso, en el contexto urbano y que se enmarcan desde el vivir bien en sus dinámicas cotidianas y que en este sentido fortalezcan el sujeto solidario, que esta cada vez más expuesto a tendencias adversas que se enmarcan como únicas y predominantes. 
Así, finalmente, se puede concluir que siguen existiendo aún grandes desafios para fortalecer la economía solidaria en Medellín, una economía que esté sustentada en otro tipo de relaciones de producción, intercambio y cooperación, y que fortalezca al sujeto solidario que supera los patrones culturales que asumen gran parte de la población, que, para este contexto en particular, sugiere ser "emprendedor". Es, como indica Acosta (2012, p. 164), necesario "construir otra lógica económica, que no radique en la ampliación permanente del consumo en función de la acumulación del capital" y que en esta misma línea tenga en cuenta las racionalidades otras de quienes habitan los barrios y tienen otra manera de vivir la vida, donde lo económico es simplemente un medio mas no un fin para ser feliz.

\section{Referencias}

Acosta, A. (2012). Buen vivir (Sumak Kawsay): una oportunidad pra imaginar nuevos mundos. Quito: Abya Yala.

Aguado, M., Calvo, D., Dessal, C., Riechmann, J., González, J. A. y Montes, C. (2012). La necesidad de repensar el bienestar humano en un mundo cambiante. Papeles de relaciones ecosociales y cambio global, 119(2), 49-76.

Alcaldía de Medellín. (2013). Modelos de operación en el proceso de desarrollo empresarial y sectorial: acompañamiento empresarial integral y fomento de la cultura solidarias de las unidades productivas asociativas $y$ agrupamientos del municipio de Medellín. Medellín: Alcaldía de Medellín.

Alcaldía de Medellín. (2011). Informe de gestión: economía solidaria. Medellín: Alcaldía de Medellín.

Alcaldía de Medellín. (s. f.). El plan de desarrollo 2008-2011: Medellín es solidaria y competitiva. Medellín: Alcaldía de Medellín.

Álzate, S. y Betancur, J. (2014). Caracterización de unidades productivas asociativas del programa de economía solidaria de la Alcaldía de Medellín. Semestre Económico, 17(0120-6346), 101-132.

Amaya, L. y Colón, C. (2013). Producción de sentido en la economía solidaria (trabajo de grado). Universidad de Antioquia, Medellín, Colombia.

Castelao, M. (2013). La economía social y solidaria en las políticas públicas nacionales y su incidencia en el territorio: alcance y perspectivas (tesis doctoral). Universidad de Buenos Aires, Buenos Aires, Argentina.

Coraggio, J. L. (2011). Economía social y solidaria, el trabajo antes que el capital. Quito: Abya-Yala.

Cordero-Ramírez, M. B. y Ortiz-Ibañez, J. A. (2012). La lógica del mercado como desreguladora de la sociedad. Otra Economía, 6(11), 125-132. doi: https://doi. org/10.4013/otra.2012.611.03

Departamento Administrativo Nacional de Estadística. (DANE). (2008). Encuesta nacional de calidad de vida. Bogotá: DANE. Recuperado de https://www.dane.gov.co/ index.php/estadisticas-por-tema/salud/calidad-de-vida-ecv/encuesta-nacional-de-calidad-de-vida-2008

Escobar, A. (2007). La invención del tercer mundo. Caracas: El perro y La Rana.

Estermann, J. (2006). Crecimiento cancerígeno versus el buen vivir. La concepción andina indígena de un desarrollo sostenible como alternativa al desarrollismo occidental. $1-14$.

Gómez, E. (2014). Decolonizar el desarrollo. Buenos Aires: Espacio Editorial

Gómez, E., Vásquez, G., Pérez, N., Tamayo, M., Gómez, C., Osorno, N. y Atehortua, O. (2009). Vivir Bien frente al desarrollo: procesos de planeacion participativa en $\mathrm{Me}$ dellin. Medellín: Pregón.

Gudynas, E. y Acosta, A. (2011). El buen vivir mas allá del desarrollo. Quehacer, (181), 70-81. Recuperado de http://www.dhl.hegoa.ehu.es/recursos/928\nhttp:// www.desco.org.pe/node/6808

Marañon-Pimentel, B. (2012). Solidaridad económica y potencialidades de transformación en América Latina: una perspectiva descolonial. Buenos Aires: CLAcso. Disponible en: http://retosalsur.org/wp-content/uploads/2013/08/ Solidaridadeconomica.pdf

Marañón -Pimentel, B. y López, D. (2014). Solidaridad económica, buen vivir y (des)colonialidad del poder. Sociedad y Discurso, 25, 153-178.

Murchio, C. (2012). Políticas públicas para la economía social y solidaria en el Gran Buenos Aires, una investigacion en el nivel local (tesis de maestría). Universidad Nacional de General Sarmiento, Argentina.

Quijano, A. (2012). ¿Bien vivir?: entre el “desarrollo”y la descolonialidad del poder. Contextualizaciones Latinoamericanas, $\operatorname{xxIV(29),~1-6.~Recuperado~de~http://~}$ www.contextualizacioneslatinoamericanas.com.mx/ pdf/Bienvivirentreeldesarrolloyladescolonialidaddelpoder_6.pdf

Razeto, L. (1993). Centralidad del trabajo y economía de solidaridad. En J.Osorio y L. Weisntein (Eds.), El corazón del arco iris. Santiago de Chile: CEPAL. 
Razeto, L. (1999). La economa solidaria: concepto, realidad y proyecto. Personas y Sociedad, 12(2). Recuperado de http://www.luisrazeto.net/content/la-econom\%C3\% ADa-solidaria-concepto-realidad-y-proyecto

Razeto, L. (2001). Desarrollo, transformación y perfeccionamiento de la economía en el tiempo. Santiago: Ediciones Universidad Boliariana.

Razeto, L. (2006, marzo). Inclusión social y economía solidaria. Inclusión social: dimensiones, retos y políticas. Caracas.

Razeto, L. (2010). ¿Qué es la economía solidaria? Papeles de Relaciones Ecosociales y Cambio Global, (110), 47-52.
Souza, M. C. (2003). Ciencia, técnica y arte: el desafío de la investigación social. En Investigación social: teoría, método, creatividad. Buenos Aires: Taylor.

Superintendencia de la Economía Solidaria. (2016). Reseña histórica. Bogotá: Supersolidaria. Recuperado de http:// www.supersolidaria.gov.co/es/nuestra-entidad/resena-historica.

Yin, R. (1994). Case Study Research: Design and Methods. Thousand Oaks: Sage. 